contains ten items of which the last three concerns tasks that some patients cannot and some do not perform. No manual for the use of ATRS has been developed. The purpose was to investigate how ATRS responds at 4, 6 and 12 months after rupture and develop a manual for the use of ATRS.

Materials and methods This study was performed as a retrospective registry study analysing prospectively gathered data from the Danish Achilles tendon Database. The data was gathered 4, 6 and 12 months after rupture. The original score based on 10 items was compared with a score based on the first 7 items adjusted to the same scale as the original score. Density- and scatterplots were made and differences between the scores were tested by t-test or Mann-Whitney U test.

Results 2790 completed ATRS scores were included. The 7item score statistically significantly overestimated the value of the 10 items score at all time points $(\mathrm{p}<0.001)$ but only at 4 months the difference was clinical relevant (9.7points).

Conclusion The ATRS cannot be recommended for use at 4 months past rupture, as the last 3 items skew the score. If the ATRS is used before 6 months the last three items could be omitted. A manual for the use of the ATRS will be developed defining how and when the score should be applied.

\section{VALIDATION OF ACHILLES TENDON LENGTH MEASURE AND ACHILLES TENDON RESTING ANGLE IN RELATION TO COPENHAGEN ACHILLES LENGTH MEASURE}

\begin{abstract}
${ }^{1}$ Maria Swennergren Hansen* ${ }^{*}{ }^{1,2}$ Morten Tange Kristensen, ${ }^{3}$ Per Hölmich, ${ }^{3}$ Kristoffer Weisskrichner Barfod. 'Physical Medicine and Rehabilitation ResearchCopenhagen (PMR-C); Department of Physical and Occupational Therapy, Copenhagen University Hospital Amager-Hvidovre, Denmark; '2Department of Orthopaedic Surgery, Copenhagen University Hospital Amager-Hvidovre, Denmark; ${ }^{3}$ Sports Orthopedic Research Center - Copenhagen (SORC-C), Department of Orthopedic Surgery, Copenhagen University Hospital Amager-Hvidovre, Denmark
\end{abstract}

\subsection{6/bjsports-2019-scandinavianabs. 21}

Introduction Elongation of the Achilles tendon after rupture is a frequent and overlooked complication. The Achilles Tendon Length Measure (ATLM) and the Achilles Tendon Resting Angle (ATRA) are indirect length measures using the resting angle of the ankle. Copenhagen Achilles Length Measure (CALM) is a direct ultrasound measure. Examination of the association of elongation to valid clinical measures of the length of the Achilles tendon are needed. The purpose was to examine the concurrent validity of ATLM and ATRA in relation to CALM within one year of rupture.

Materials and methods The study was performed as a validity study. Data were collected from patients included in a randomized controlled trial. Mixed linear regression, controlling for time after injury, age and gender, was performed investigating the three models (dependent-independent): CALM-ATRA, CALM-ATLM and ATRA-ATLM.

Results 130 patients were included (23 women, 107 men) mean age 41.8 years (SD 10.5). All three regression models demonstrated a statistical significant $(\mathrm{p}<0.01)$ linear relationship. For each degree ATRA increased, CALM increased with $0.39 \mathrm{~mm}$. For each $\mathrm{cm}$ ATLM increased, CALM increased with $1.7 \mathrm{~mm}$. For each $\mathrm{cm}$ ATLM increase, ATRA increase with 1,6 degrees.

Conclusion ATRA and ATLM were found to have a linear relationship to CALM and seems valid as surrogate measurements for the assessment of tendon elongation after an Achilles tendon rupture.

\section{RUNNING A MARATHON - THE EFFECT ON ACHILLES TENDON STRUCTURE}

'Lucas Maciel Rabello*, ${ }^{1,2}$ Mathijs van Ark, ${ }^{1}$ Sophie Albers, ${ }^{3}$ Ron L Diercks, ${ }^{1} J o h a n n e s$ Zwerver, ${ }^{1,3}$ Inge van den Akker-Scheek. 'University of Groningen, University Medical Center Groningen, Department of Sports and Exercise Medicine, Netherlands; ${ }^{2}$ Hanze University of Applied Sciences Groningen, Department of Physiotherapy, Netherlands; ${ }^{3}$ University of Groningen, University Medical Center Groningen, Department of Orthopedics, Netherlands

\subsection{6/bjsports-2019-scandinavianabs.22}

Introduction More than half a million athletes were enrolled in a marathon run during the last years. During running the Achilles tendon is submitted to high loads, and multiple tensile strain cycles. There are no studies investigating the effect of a marathon in the structure of the Achilles tendon. Thus, the aim of this study was to investigate the short-term response of the Achilles tendon structure after running a marathon.

Materials and methods Ten male non-elite runners who participated in a marathon were included. Tendon structure was assessed before, 2 days and 7 days after a marathon using the ultrasound tissue characterization (UTC), an imaging tool which quantifies tendon organization dividing the structure into four different echo types (I-IV).

Results Two days after a marathon, no significant changes in tendon structure were observed. However, after 7 days both insertional and midportion structure changed significantly. At the insertion of the tendon there was a significant decrease in the percentage of echo types I, III and IV and a significant increase in the percentage of echo type II. The midportion of the tendon showed a significant decrease in the percentage of echo types III and IV and a significant increase in the percentage of echo type II.

Conclusion We observed that the effects of running a marathon on the Achilles tendon structure occured 7 days after the event. In a population of runners without tendon injuries, there seems to be a positive adaptation of the tendon to the load after running a marathon.

\section{EVALUATION OF IN-EAR SENSOR SYSTEMS FOR QUANTIFYING HEAD IMPACT EXPOSURE IN YOUTH FOOTBALL}

${ }^{1}$ Stian Bahr Sandmo*, 2,3,4 Andrew S Mclntosh, ${ }^{1}$ Thor Einar Andersen, ${ }^{5,6}$ Inga K Koerte, ${ }^{1}$ Roald Bahr. 'Department of Sports Medicine, Oslo Sports Trauma Research Center, Norwegian School of Sport Sciences, Norway; ${ }^{2}$ Federation University Australia, Australia; ${ }^{3}$ Monash University Accident Research Centre, Monash University, Australia; ${ }^{4}$ McIntosh Consultancy and Research, Australia; ${ }^{5}$ Department of Child and Adolescent Psychiatry, Psychosomatic and Psychotherapy, Ludwig-Maximilian University, Germany; ${ }^{6}$ Department of Psychiatry, Pscyhiatry Neuroimaging Laboratory, Brigham and Women's Hospital, Harvard Medical School, USA

\subsection{6/bjsports-2019-scandinavianabs.23}

Introduction Wearable sensor systems may be useful for measuring head-impact exposure. Here, we tested the validity of in-ear sensors developed to improve head coupling.

Methods First, the sensor was mounted to a Hybrid III headform (HIII) and impacted with a linear impactor or football. Peak linear acceleration (PLA), peak rotational acceleration (PRA) and peak rotational velocity (PRV) were obtained from both systems; random and systematic error were calculated using HIII as reference. Then, six youth football players wore sensors and performed a structured training protocol 
including heading and non-heading exercises; they also completed two regular football sessions. For each accelerative event recorded, PLA, PRA and PRV outputs were compared to video recordings. Receiver operating characteristic curves were used to determine the sensor's discriminatory capacity in both on-field settings, determining cut-off values for predicting outcomes.

Results For the laboratory tests, the random error was $11 \%$ for PLA, 20\% for PRA and 5\% for PRV, respectively; the systematic error was $11 \%, 19 \%$ and $5 \%$. For the structured training protocol, heading events yielded higher absolute values $(\mathrm{PLA}=15.6 \pm 11.8 \mathrm{~g}$ ) than non-heading events (PLA=4.6 $\pm 1.2 \mathrm{~g}$ ); the area under the curve (AUC) was 0.98 for PLA. In regular training sessions, AUC was $>0.99$ for PLA. A $9 \mathrm{~g}$ cut-off value yielded a positive predictive value of $100 \%$ in the structured training protocol, compared to only $65 \%$ in regular football sessions.

Conclusion The sensor displayed systematic overestimation with considerable random error. Despite excellent on-field accuracy for discriminating head-impacts from other accelerative events, secondary means of verifying events are still necessary.

\section{EVALUATION OF IN-EAR SENSOR SYSTEMS FOR QUANTIFYING HEAD IMPACT EXPOSURE IN YOUTH FOOTBALL}

${ }^{1}$ Stian Bahr Sandmo*, 2,3,4Andrew S Mclntosh, 'Thor Einar Andersen, 5,6Inga K Koerte, ${ }^{1}$ Roald Bahr. 'Department of Sports Medicine, Oslo Sports Trauma Research Center, Norwegian School of Sport Sciences, Norway; ${ }^{2}$ Federation University Australia, Australia; ${ }^{3}$ Monash University Accident Research Centre, Monash University, Australia; ${ }^{4}$ McIntosh Consultancy and Research, Australia; ${ }^{5}$ Department of Child and Adolescent Psychiatry, Psychosomatic and Psychotherapy, Ludwig-Maximilian University, Germany; ${ }^{6}$ Department of Psychiatry, Pscyhiatry Neuroimaging Laboratory, Brigham and Women's Hospital, Harvard Medical School, USA

\subsection{6/bjsports-2019-scandinavianabs.24}

Introduction Wearable sensor systems may be useful for measuring head-impact exposure. Here, we tested the validity of in-ear sensors developed to improve head coupling.

Methods First, the sensor was mounted to a Hybrid III headform (HIII) and impacted with a linear impactor or football. Peak linear acceleration (PLA), peak rotational acceleration (PRA) and peak rotational velocity (PRV) were obtained from both systems; random and systematic error were calculated using HIII as reference. Then, six youth football players wore sensors and performed a structured training protocol including heading and non-heading exercises; they also completed two regular football sessions. For each accelerative event recorded, PLA, PRA and PRV outputs were compared to video recordings. Receiver operating characteristic curves were used to determine the sensor's discriminatory capacity in both on-field settings, determining cut-off values for predicting outcomes.

Results For the laboratory tests, the random error was $11 \%$ for PLA, 20\% for PRA and 5\% for PRV, respectively; the systematic error was 11\%, 19\% and 5\%. For the structured training protocol, heading events yielded higher absolute values $(\mathrm{PLA}=15.6 \pm 11.8 \mathrm{~g}$ ) than non-heading events (PLA=4.6 $\pm 1.2 \mathrm{~g}$ ); the area under the curve (AUC) was 0.98 for PLA. In regular training sessions, AUC was $>0.99$ for PLA. A $9 \mathrm{~g}$ cut-off value yielded a positive predictive value of $100 \%$ in the structured training protocol, compared to only $65 \%$ in regular football sessions.
Conclusion The sensor displayed systematic overestimation with considerable random error. Despite excellent on-field accuracy for discriminating head-impacts from other accelerative events, secondary means of verifying events are still necessary.

\section{INCREASED TTTG MEASURED ON AXIAL MRI. IS IT DUE TO LATERALIZATION OF THE TIBIAL-TUBERCLE OR MEDIALIZATION OF THE TROCHLEAR-GROOVE?}

${ }^{1}$ Mathias Paiva, ${ }^{2}$ Lars Blønd, ${ }^{1}$ Per Hölmich, ${ }^{1}$ Kristoffer Barfod*. ${ }^{1}$ Sports Orthopedic Research Center Copenhagen (SORC-C), Department of Orthopedic, Danmark; 'Zealand University Hospital, Køge, Danmark

\subsection{6/bjsports-2019-scandinavianabs.25}

Introduction To investigate if increased Tibial tubercle - Trochlear Groove distance (TT-TG) measured on axial MRI is due to lateralization of the tibial tubercle or medialization of the trochlear groove.

Methods 65 knees (28 normal (NK), 25 with trochlear dysplasia (TD) and 12 with patellar dislocation without TD (PD)) were examined. The medial border of the posterior cruciate ligament (PCL) was chosen as the central anatomical landmark. The distance from the tibial tubercle (TT) to PCL (TTPCL) was measured to examine the lateralization of the tibial tubercle. The distance from the trochlear groove to PCL (TGPCL) was measured to examine the medialization of the trochlear groove. Between group differences was investigated by use of one-way ANOVA.

Results The mean (SD) values for TT-TG were $8.5 \mathrm{~mm}$ (3.6) for NK, $11.4 \mathrm{~mm} \mathrm{(6.2)} \mathrm{for} \mathrm{PD} \mathrm{and} 17.1 \mathrm{~mm} \mathrm{(4.8)}$ in the TD group $(\mathrm{p}<0.01)$. The mean $(\mathrm{SD})$ values for TT-PCL were $19.5 \mathrm{~mm}$ (4.2) for NK, $17.0 \mathrm{~mm} \mathrm{(5.0)} \mathrm{for} \mathrm{PD} \mathrm{and} 20.2 \mathrm{~mm}$ (5.0) in the TD group $(p=0.10)$. The mean (SD) values for TG-PCL were $10.5 \mathrm{~mm}$ (3.7) for NK, $5.8 \mathrm{~mm} \mathrm{(4.9)} \mathrm{for} \mathrm{PD}$ and $3.9 \mathrm{~mm}(3.9)$ in the dysplastic group $(\mathrm{p}<0.01)$.

Conclusion TD knees had increased TT-TG compared to NK and PD. The TT-PCL distance did not differ significantly between groups, whereas the TG-PCL distance declined with increased TT-TG. The present results indicate that increased TT-TG is due to medialization of the trochlear groove and not lateralization of the tibial tubercle.

\section{AFTER SIX-WEEKS OF INTENSIVE BALLET REHEARSALS CHANGES IN THE ACHILLES TENDONS STRUCTURE APPEAR WHEREAS SELF-REPORTED SEVERITY SCORES REMAIN THE SAME}

\begin{abstract}
${ }^{1,2}$ Charlotte Anker-petersen*, ${ }^{2}$ Kristian Thorborg, ${ }^{3}$ Jarrod Antflick, ${ }^{4}$ Henrik Aagaard, ${ }^{3}$ Chris Myers, ${ }^{2}$ Anders Ploug Boesen, ${ }^{2}$ Per Hølmich, ${ }^{1}$ Birgit Juul-Kristensen. ${ }^{1}$ Department of Sports Science and Clinical Biomechanics, University of Southern Denmark, Denmark; ${ }^{2}$ Sports Orthopedic Research Center - Copenhagen; Department of Orthopedic Surgery, Amager-Hvidovre Hospital, Denmark; ${ }^{3}$ Tendon Performance, UK; ${ }^{4}$ Department of Orthopedic Surgery, Sjaelland University Hospital, Denmark
\end{abstract}

\subsection{6/bjsports-2019-scandinavianabs.26}

Introduction The aim was to study whether six weeks of intensive dance exposure (Swan Lake rehearsals) is a significant contributor to structural changes, symptoms, clinical signs and pain in the Achilles tendon (AT).

Materials and methods Ballet dancers from The Royal Danish Ballet Company (aged 18-41) were invited $(n=79)$ of which 